\title{
Stress-induced effects in light scattering by plasmons in $p$-type germanium
}

\author{
V.N. Poroshin, A.V. Gaydar, A.A. Abramov*, V.N. Tulupenko* \\ Institute of Physics, NAS of Ukraine, 46 prospect Nauki, 03028 Kyiv, Ukraine \\ Fax:+380 (44) 2651589,E-mail: poroshin@iop.kiev.ua \\ *Donbass State Machine-building Academy, 72 Shkadinov Str., 84313 Kramatorsk, Ukraine \\ E-mail: tvn@laser.donetsk.ua
}

\begin{abstract}
Infrared light scattering by plasmons in $p$-Ge has been studied under uniaxial stress along the [110] axis with polarization of incident light parallel to the stress direction. It is found that the deformation of the crystal results in an increase of the plasma frequency and lowering the asymmetry of the line by plasma scattering. These effects are explained by taking into account the change of contribution to the dielectric constant caused by the intra- and intersubband transitions as a consequence of variation of the energy band of $p$-Ge related with deformation.
\end{abstract}

Keywords: light scattering, plasmon, plasma frequency, stress, dielectric constant, germanium.

Paper received 13.10.03; accepted for publication 11.12.03.

\section{Introduction}

Uniaxial deformation of cubic semiconductors causes substantial changes of valence band spectrum of the holes [1]. Degeneracy of the light and heavy hole subbands in the point of maximum is removed and their shape changes due to reduction of crystal symmetry. This effect results in changes of various crystal properties related to free carriers.

Effects of uniaxial elastic deformation on electric conduction, galvanomagnetic and thermomagnetic phenomena have been well investigated for various $p$-type semiconductors (see, for example [2]). A variation of optical properties for semiconductors with a degenerate valence band in the IR spectral range under uniaxial stress has been observed. In particular, anisotropy of such optical crystal characteristics as the refraction index and absorption coefficient, variation of magnitude and spectral dependencies of linear and non-linear light absorption coefficients due to hole intersubband transitions were found [3-5]. Besides, a number of phenomena related to intersubband hole transitions near the subband maximum appeared in the crystals under deformation has been considered theoretically. In particular, resonance light absorption and corresponding spectral dependencies of the photoconductivity and photon drag effect of holes, the
Raman scattering of light with frequency shift by the energy of subband maximum splitting were considered [6-8].

The present paper reports on observation and investigation of new features in light scattering by collective oscillations of free carriers, referred to as plasmons, in pGe crystals under uniaxial elastic compressive stress.

\section{Experimental procedure}

Light scattering by plasmons was investigated in germanium samples with acceptor $(\mathrm{Ga})$ concentrations of $2.3 \cdot 10^{17} \mathrm{~cm}^{-3}$ and $4.5 \cdot 10^{17} \mathrm{~cm}^{-3}$. The concentration of free carriers in the samples was determined using the Halleffect.

The samples were cut as rectangular parallelepipeds with all faces parallel to the crystallographic plane (110). The uniaxial compression within the range of 0 to $2.5 \mathrm{kbar}$ was applied to a sample along the crystallographic direction [110].

We used the infrared (IR) light of the high-stable $\mathrm{CO}_{2}-$ laser with $\lambda=10.6 \mu \mathrm{m}$ as exciting radiation. The laser pulse duration was $300 \mathrm{~ns}$ and the repetition frequency $20 \mathrm{~Hz}$. The incident IR light was polarized along the direction of the applied stress.

The light scattering measurements were performed at $80 \mathrm{~K}$ in the backscattering geometry. The scattered light 


\section{V.N. Poroshin et al.: Stress-induced effects in light scattering by plasmons...}

was analyzed by an IR monochromator using a cooled $\mathrm{CdHgTe}$ photoconductor cell and computer-controlled system of pulse accumulation. In addition, $\mathrm{SF}_{6}$ gas filter was mounted in front of the input slit of the monochromator. It enabled to avoid transmission of the light scattered elastically in the crystal and elements of the optical facilities into the recording system (the ratio of the intensities of the incident and scattered light was of the order of $10^{12}$ ).

The incident laser pulse intensity at the crystal surface was $300 \mathrm{~kW} / \mathrm{cm}^{2}$. Inelastically scattered light was of the order $10^{-9}$ to $10^{-8} \mathrm{~W}$. At the temperature under study all the impurity centers in germanium were ionized and carriers were in the valence band.

\section{Experimental results}

Fig.1 presents typical spectra of inelastic light scattering (Raman spectra) for the $p$-Ge samples measured in the range from -150 to $+150 \mathrm{~cm}^{-1}$ under uniaxial stress $X$.

In the absence of stress, Raman spectra coincide with those measured earlier [9]. The line due to light scattering by collective excitations of carriers (plasmons) was observed on the background of the Stokes side of the wide (from 0 to $\pm 150 \mathrm{~cm}^{-1}$ ) asymmetric band caused by quasielastic scattering connected with single-particle unscreened excitations of free holes. The latter are fluctuations of the quadrupole momentum of holes originating from
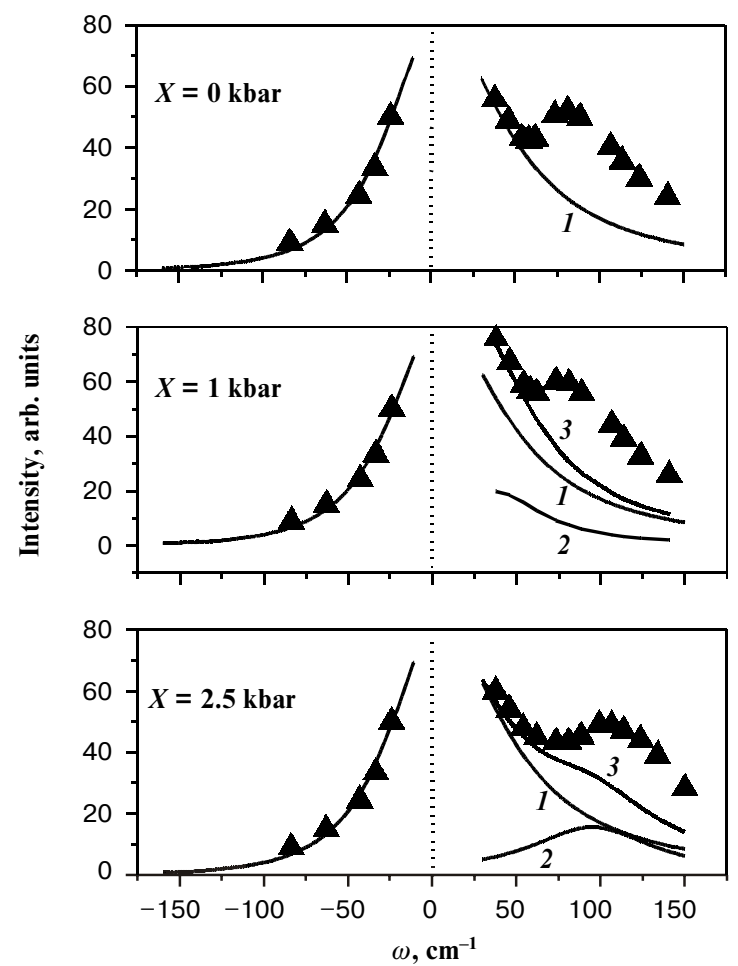

Fig. 1. Typical electronic Raman spectra of $p-\mathrm{Ge}$ at $80 \mathrm{~K}$ for different values of uniaxial stress along [110]. The solid lines show the calculated spectra for intrasubband (1), intersubband (2) and total (3) electronic scattering. intrasubband transitions. The plasmon line was identified in [9] by the dependence of the maximum frequency on the carrier concentration and by the dependence of the scattering intensity on the mutual polarization directions of the incident $\mathbf{e}_{\mathbf{i}}$ and scattered $\mathbf{e}_{\mathbf{s}}$ light (the line was observed only in the case of $\mathbf{e}_{\mathbf{i}} / / \mathbf{e}_{\mathbf{s}}$ ).

Fig. 2 shows the reduced Raman spectrum for the nonstressed $\mathrm{p}-\mathrm{Ge}$ obtained by subtraction of the intrasubband scattering from the complete spectrum. The intrasubband scattering band contour was fitted by the following expression

$$
\begin{aligned}
& I(\omega) \sim F(\omega) \frac{A}{\omega^{2}+A^{2}}, \\
& F(\omega)=\hbar \omega /\{1-\exp (-\hbar \omega / k T)\}
\end{aligned}
$$

which well describes spectra of light scattered by singleparticle excitations in the case of collisions of carriers with crystal lattice defects, as shown in [10]. The magnitude of fitting parameter A was selected with regard to a best agreement between the calculated curve and experimental Raman spectrum in the frequency range from $150 \mathrm{~cm}^{-1}$ to about $40 \mathrm{~cm}^{-1}$, where scattering by plasmons is in fact absent. It is seen from Fig. 2 that the plasmon line for the non-stressed crystal is wide and asymmetric.

The uniaxial compression applied to the $p$-Ge crystal led to changes of the Stokes part in the observed Raman spectrum, in particular, to an increase of scattered light

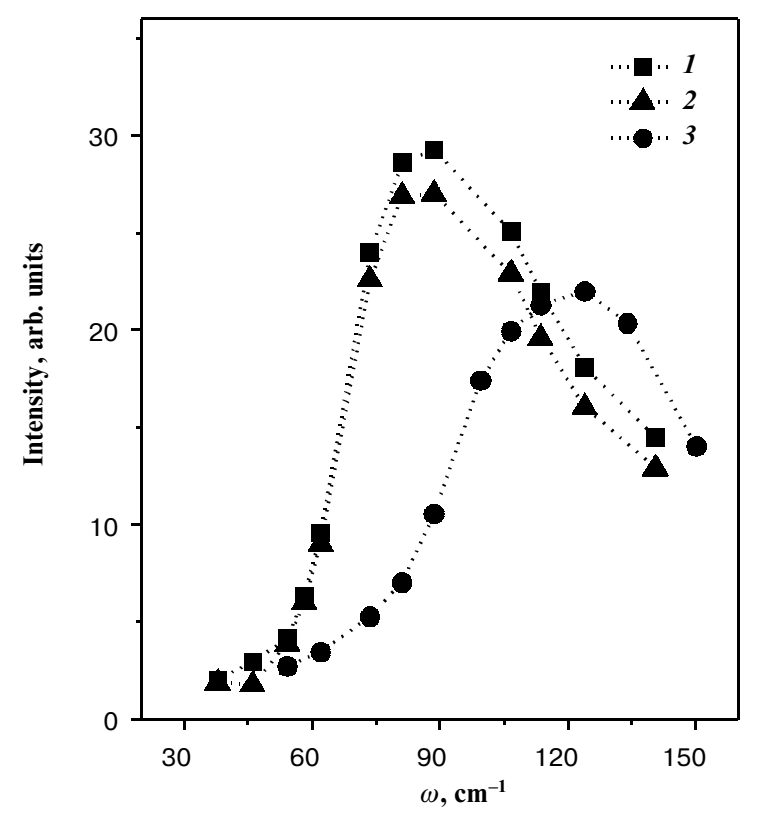

Fig. 2. Plasmon line for different stress values: $1-0,2-1,3-$ 2.5 kbar. The dots represent experimental data while the solid line is a fit using (6). $N=4.5 \times 10^{17} \mathrm{~cm}^{-3}$. 


\section{V.N. Poroshin et al.: Stress-induced effects in light scattering by plasmons...}

intensity and to a change of the plasmon line shape. Besides, at pressure $X>1 \mathrm{kbar}$ maximum frequency of the line varies. At the same time, the anti-Stokes part of the spectrum, which is determined basically by light scattering on the single-particle excitations of free carriers, did not change under uniaxial stress up to its maximum magnitude used in experiment $X=2.5 \mathrm{kbar}$.

On the one hand, the changes observed in Raman spectra are related to changes in light scattering by plasmons under the crystal stress, and, on the other hand, to an appearance of light scattering due to hole quadrupole momentum fluctuations during transitions between the valence subbands splitted by the stress near their maxima. The splitting subband energy at the maximum point, $E_{0}$, is determined by the applied uniaxial stress: $E_{0}=\chi X$ and changes from 0 to $90 \mathrm{~cm}^{-1}$ with increase of $\mathrm{X}$ from 0 to $2.5 \mathrm{kbar}$ (according to [11], $\chi=39 \mathrm{~cm}^{-1} / \mathrm{kbar}$ for $X \|$ [110]).

We observed intersubband scattering of IR light in uniaxially stressed samples of $p$-Ge with the carrier concentration from $5 \cdot 10^{15} \mathrm{~cm}^{-3}$ to $3 \cdot 10^{16} \mathrm{~cm}^{-3}$ when the plasmon line did not fall in the recorded spectral range [12]. The Raman line was observed at the frequency $\omega_{\max }=$ $=E_{0} / \hbar$. In the range of the applied pressures under study, the line had the Lorentz shape, and its width was proportional to the pressure $X$ and varied from about $60 \mathrm{~cm}^{-1}$ to $78 \mathrm{~cm}^{-1}$ with $X$ varying from $1 \mathrm{kbar}$ to $2.5 \mathrm{kbar}$. At the same time, the line contour area that determines the integral cross section remains constant.

The calculated lines of intersubband light scattering at $X=1$ and $2.5 \mathrm{kbar}$ are shown in Fig. 1 . The sum of both intra and intersubband components of light scattering in the observed Raman spectrum is shown here, too. The intensity and shape of the intrasubband light scattering band were taken to be the same in the calculations for both stressed and non-stressed crystals. For the intersubband scattering, the intensity of the line with the known width and maximum frequency was fitted to obtain a good agreement between the calculated total single-particle spectrum and the Stokes Raman spectrum observed experimentally for stressed samples at low frequencies (below approximately $50 \mathrm{~cm}^{-1}$ ) where light scattering by plasmons is absent.

After subtraction of the single-particle scattering component from the observed Raman spectrum, we obtain the plasmon line. This line is shown in Fig. 2 for different magnitudes of applied stress. As seen from Fig. 2, under uniaxial stress of the p-Ge crystal, the line asymmetry for plasma light scattering decreases. The line maximum frequency that defines the plasma frequency of carriers $\omega_{p}$ does not vary indeed under the applied stress $X$ up to $X=1 \mathrm{kbar}$. At $X>1 \mathrm{kbar} \omega_{p}$ increases with growing stress $X$. Fig. 3 illustrates the dependence of the plasma frequency versus stress for the $p$-Ge samples. At some given magnitude of the stress, the magnitude of the plasma frequency change differs for samples with different concentrations of free carriers. For example, at $X=2.5 \mathrm{kbar}$, it is about 20 and $36 \mathrm{~cm}^{-1}$ for $N=2.3 \cdot 10^{17}$ and $N=4.5 \cdot 10^{17} \mathrm{~cm}^{-3}$, respectively.

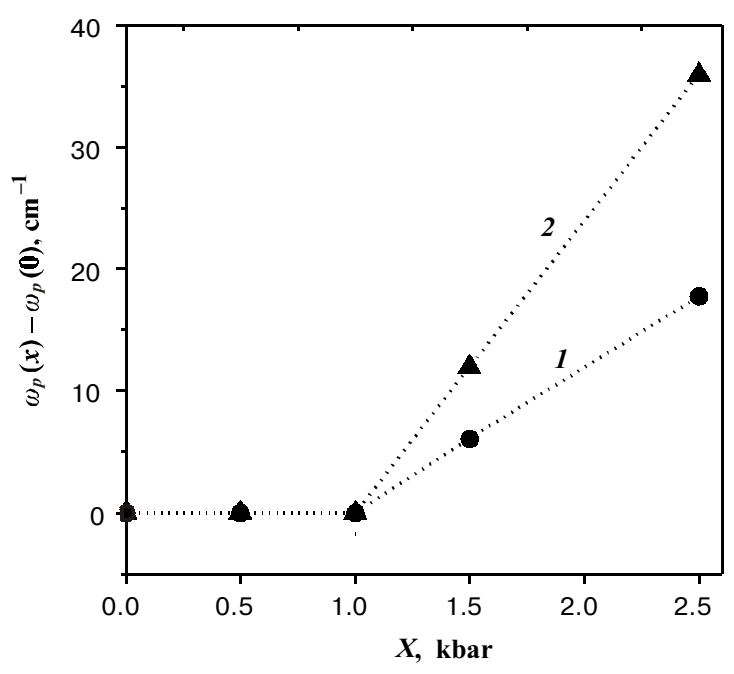

Fig. 3. Plasma frequency vs stress in $p$-Ge with different hole concentration: $1-N=2.3 \times 10^{17}, 2-N=4.5 \times 10^{17} \mathrm{~cm}^{-3}$.

\section{Discussion}

The plasma frequency of free carriers and the line shape for light scattered by plasmon are determined by the frequency dependence of the dielectric constant of the crystal. For cubic $p$-type semiconductors, the dielectric constant may be written as

$\varepsilon=\varepsilon_{0}+\varepsilon_{\text {intra }}+\varepsilon_{\text {inter }}$,

where $\varepsilon_{0}$ is the lattice dielectric constant, $\varepsilon_{\text {intra }}$ describes the dielectric constant components due to virtual intrasubband electron transitions, the last term $\varepsilon_{\text {inter }}$ is the intersubband term .

Therefore, the observed behavior of plasma frequency and the plasmon line shape in the stressed $p$-Ge has to be related to the change of the dielectric constant caused by changes in the energy band spectrum of carriers on account of deformation. The energy gap arising by the deformation increases with growing stress $X$ applied to a crystal. The subbands change their shape so that with growing stress the warped surface of a constant energy becomes an ellipse compressed or elongated along the deformation axis. As a consequence, the dielectric constant and, hence, plasma frequency as well as the line shape for light scattered by plasmons become dependent on the magnitude of applied stress.

Note that a uniaxial crystal deformation violates the cubic symmetry in distribution of free carriers in the $\mathbf{k}$ space. Hence, the dielectric constant becomes anisotropic. For the direction of uniaxial compressive stress of the Ge crystals along [110] one of the main axes of the dielectric constant tensor coincides with this direction, while two other are directed along [110] and [001]. All three main values of the tensor (longitudinal $\varepsilon_{\| \mid}$and transverse $\varepsilon_{\perp 1}$, $\varepsilon_{\perp 2}$ in regard to deformation direction) are non-zero. Since the polarization direction of incident IR light in our experiment coincides with the deformation direction 


\section{V.N. Poroshin et al.: Stress-induced effects in light scattering by plasmons...}

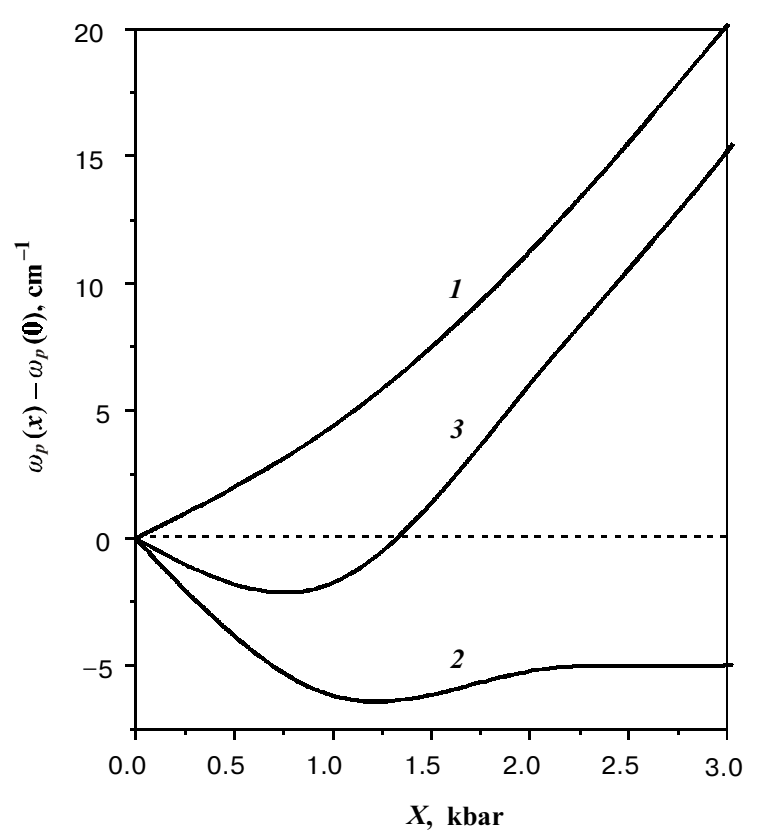

Fig. 4. Plasma frequency change in $p$-Ge $\left(\mathrm{N}=4.5 * 10^{17} \mathrm{~cm}^{-3}\right)$ vs stress. Contributions to the dielectric constant of intrasubband (1), intersubband (2) and both types (3) of electron transitions.

the measured plasma frequency and spectral plasmon line dependence are defined by the longitudinal component of the dielectric constant tensor.

The dielectric constant of uniaxially stressed p-type semiconductors was considered in [6-7]. Its real part is given by:

$$
\begin{aligned}
& \varepsilon_{\|}^{\prime}(\omega)=\varepsilon_{0}-\frac{4 \pi e^{2}}{\left(\omega^{2}+\Gamma^{2}\right)} \frac{2}{V} \sum_{\mathbf{p}}\left[f_{+} \frac{\partial^{2} E_{+}}{\partial p_{\|}^{2}}+f_{-} \frac{\partial^{2} E_{-}}{\partial p_{\|}^{2}}\right] \\
& -\frac{4 \pi e^{2}}{\omega^{2} V} \sum_{\mathbf{p}} \mathrm{M}_{\|}(\mathbf{p})\left(f_{-}-f_{+}\right) \frac{2}{E_{-}-E_{+}} \frac{(\hbar \omega)^{2}}{\left(E_{-}-E_{+}\right)^{2}-(\hbar \omega)^{2}}
\end{aligned}
$$

where $E_{ \pm}$is the energy of carriers in split subbands, $\mathrm{f}_{ \pm}$is their Fermi function, $M_{\|}(p)$ is the matrix element of intersubband direct transitions, $\Gamma$ is the effective frequency of carrier collision with lattice defects, which is assumed to be the same for different subbands. The second and third terms in (3) describe the components of virtual intra- and intersubband transitions, respectively.

The matrix element of intersubband transitions in stressed crystals is given in [6] without taking into account valence band warping and anisotropy of the elastic properties of crystals

$$
\begin{aligned}
& \mathrm{M}_{\|}(\mathbf{p})=\frac{6(2 \gamma / m)^{2}}{\left(E_{+}-E_{-}\right)^{2}} p_{\perp}^{2} \times \\
& \times\left[\left(\frac{\gamma p^{2}}{m}\right)^{2}-\frac{E_{0} \gamma}{2 m}\left(p^{2}+p_{z}^{2}\right)+\frac{E_{0}^{2}}{4}\right] .
\end{aligned}
$$

The dependence of carrier energy on their momentum $\mathbf{p}$ in the subbands is defined as

$$
E_{ \pm}=\frac{\gamma_{1} p^{2}}{2 m} \pm \sqrt{\left(\frac{\gamma p^{2}}{m}\right)^{2}+E_{0} \frac{\gamma p^{2}}{m} \mathrm{P}_{z}\left(\frac{p_{z}}{p}\right)+\frac{E_{0}^{2}}{4}}
$$

where $\gamma_{1}$ and $\gamma$ are the Luttinger parameters of the isotropic approximation, $p_{z}$ is the projection of carrier momentum on the direction of stress, $P_{2}$ is the second Legendre polynomial, $E_{0}$ is the splitting energy of the subband maxima, which depends on the applied stress.

Solution of the equation $\varepsilon_{\|}^{\prime}\left(\omega_{p}\right)=0$ gives the dependence of the plasma frequency $\omega_{p}$ on the magnitude of uniaxial stress. The variation of $\omega_{p}$ is negligible up to 1 kbar. An increase of $\omega_{p}$ occurs at $X>1$ kbar. Fig. 5 illustrates the dependence of $\omega_{p}$ on X calculated for $p$-Ge with the hole concentration $N=4.5 \cdot 10^{17} \mathrm{~cm}^{-3}$. These calculations do not take into account any collisions of carriers $(\Gamma=0)$. The following parameters of Ge were used: $\varepsilon_{0}=16, \gamma_{1}=13.35$ and $\gamma=5.25$ [11].

Such a dependence of $\omega_{p}$ on $\mathrm{X}$ is explained by the fact that changes of the dielectric constant components for intra- $\varepsilon_{\text {intra }}$ and intersubband $\varepsilon_{\text {inter }}$ transitions caused by the crystal deformation have different impact on the plasma frequency (curves 1 and 2 in Fig. 5). Thus, change of the intrasubband component $\varepsilon_{\text {intra }}$ results in an increase of the plasma frequency $\delta \omega_{\text {intra }}=\omega_{\text {intra }}(X)-\omega_{\text {intra }}(0)>0$, the increase being stronger at higher $X$. It is basically connected with a decrease of the effective mass of holes in the lower subband under deformation and the transitions of carriers to it from the upper split-off subband. The plasma frequency change related to intersubband transitions is negative $\left(\delta \omega_{\text {inter }}<0\right)$. It is maximal at the pressure about $1 \mathrm{kbar}$. That is connected with the contribution to the dielectric constant of the carrier transitions between

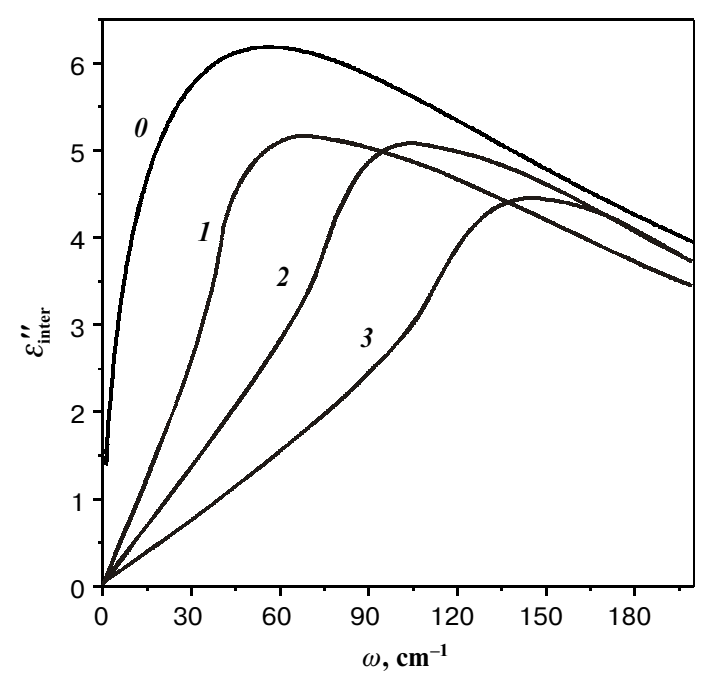

Fig. 5. Intersubband contribution to the imaginary part of the dielectric constant of $-\mathrm{Ge}$ with the hole concentration $N=$ $=4.5 \times 10^{17} \mathrm{~cm}^{-3}$ for various uniaxial stress values: $0-0 \mathrm{kbar}, 1-1$, $2-2,3-3$ kbar. 


\section{V.N. Poroshin et al.: Stress-induced effects in light scattering by plasmons...}

the split subbands near the maxima. The magnitude of this contribution reaches a maximum in the range of frequencies corresponding to the sum of splitting energy at the subband maxima and average energy of carriers, as shown in [6,7]. At $X<1$ kbar the plasma frequency changes related to the intrasubband transitions $\delta \omega_{\text {intra }}$ are compensated by its variation due to intersubband

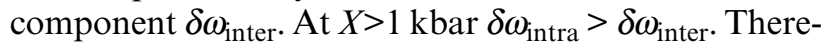
fore, the plasma frequency increases with the growing pressure applied to the crystal.

The calculated dependence of the plasma frequency on the applied pressure is similar to that obtained experimentally for $p$-Ge. Discrepancy between calculated and experimental values $\delta \omega_{p}$ is caused by the fact that the calculations did not take into account the warping of the valence band and the anisotropy of elastic properties of germanium. These factors taken into account have an impact on the magnitude of dielectric constant components due to intra and intersubband transitions and, consequently, on the plasma frequency, as shown in [7]. However, it does not change its dependence on the applied pressure.

Let us consider now the influence of a uniaxial stress on the plasmon line shape. The spectral dependence of the cross section for light scattering by plasmons, which defines the line shape, is given by:

$\sigma(\omega) \sim \operatorname{Im}[-1 / \varepsilon(\omega)]$

In the non-stressed $p$-Ge crystals the asymmetry of the plasmon line is caused by the component of intersubband transitions in the imaginary part of the dielectric constant $\varepsilon^{\prime \prime}{ }_{\text {inter }}$ [9]. At the same time, the line width is determined also by collisions of carriers, which occur mainly with impurities in the crystals under study. Therefore, changes in the line shape under uniaxial stress must be related to changes in the collision frequency $\Gamma$ and the frequency dependence of $\varepsilon^{\prime \prime}$ inter.

The imaginary part of the dielectric constant in the stressed p-type crystals may be written as [6]

$$
\begin{aligned}
& \varepsilon_{\|}^{\prime \prime}(\omega)=\frac{4 \pi e^{2} \Gamma}{\omega\left(\omega^{2}+\Gamma^{2}\right)} \frac{2}{V} \sum_{\mathbf{p}}\left[f_{+} \frac{\partial^{2} E_{+}}{\partial p_{\|}^{2}}+f_{-} \frac{\partial^{2} E_{-}}{\partial p_{\|}^{2}}\right]+ \\
& +\frac{4 \pi^{2} e^{2}}{\omega^{2} V} \sum_{\mathbf{p}} \mathrm{M}_{\|}(\mathbf{p})\left(f_{+}-f_{-}\right) \delta\left(E_{+}-E_{-}\right)
\end{aligned}
$$

Changes in the frequency dependence of $\varepsilon^{\prime \prime}{ }_{\text {inter }}$ under stress calculated according to (7) are shown in Fig. 4. As can be seen, with an increasing uniaxial pressure, the curve $\varepsilon^{\prime \prime}{ }_{\text {inter }}(\omega)$ shifts to a higher frequencies and the dielectric constant component due to intersubband transitions actually decreases in the spectral range containing the plasmon line.

The dashed line in Fig. 2 shows the spectral dependence of the plasma light scattering in $\mathrm{p}-\mathrm{Ge}$ calculated for various magnitudes of the applied uniaxial stress and taking into account the changes of $\varepsilon^{\prime \prime}$ inter $(\omega)$. The colli- sion frequency of holes was chosen to obtain the best agreement between the theoretical curve and experimental data. In the pressure range $0<X \leq 1.5 \mathrm{kbar}$, the collision frequency of holes occurred to be close to that obtained from the carrier mobility measured under dc electric field in non-stressed crystals $\Gamma(0)$. However, at $X=2.5 \mathrm{kbar}$ the collision frequency of carriers turned out to be almost 4 times as large as $\Gamma(0)$. It gives evidence of the enhanced scattering of carriers by impurities in the pressure range from 1.5 to $2.5 \mathrm{kbar}$. From our point of view, the effect is caused by the transformation of the localized acceptor states into resonance (quasi-local) states. Existence of these states in uniaxially stressed Ge was experimentally evidenced, for example, in [14, 15]. Such states arise due to stresses in the crystal leading to the splitting of impurity levels. One of the two split impurity levels at some magnitude of the deformation shifts into continuous spectrum of energy of the lower valence subband and becomes, therefore, quasi-local. At the same time, hole scattering by impurities increases sharply when the quasi-local state energy reaches the average energy of holes (resonance scattering) $[16,17]$.

The resonance states in uniaxially stressed germanium were calculated in many investigations basing on various approximations for the impurity potential (for example, [16-19]). The obtained values of valence subband splitting energy due to deformation, in the case when the main acceptor state becomes resonance, considerably differ from each other. For Ga impurity they lie within the range from 8 to $16 \mathrm{meV}$ that corresponds to pressures between 1.8 to $3.6 \mathrm{kbar}$ for stresses along [110]. The magnitude of uniaxial stress, for which an increase of hole scattering by impurities occurs, lies in this pressure range.

The authors are grateful to F.T. Vasko and O.G. Sarbey for the discussion of results and to V.M. Vasetski for his assistance in carrying out the experiments.

\section{References}

1. G.L. Bir, G.E. Pikus. Symmetry and strain-induced effects in semiconductors. Eds. J.Wiley\&Sons, New York-Toronto (1974).

2. P.I.Baranskii, V.P.Klochkov, I.V.Potikevich. Semiconductor Electronics. Naukova Dumka, Kiev (1975).

3. S. Riskaer, L. Balslev, Interband piezo-birefringence in germanium // Phys. Lett. 21(1), pp. 16-18 (1966).

4. R.B. James, D.L. Smith, Effect of unixial stress on the saturation of intervalence-band absorption in p-type Ge // Phys. Rev. B28(2) pp. 586-592 (1983).

5. S.M. Kelso, Energy- and stress-dependent hole masses in germanium and silicon // Phys.Rev. 25B(2), pp. 1116-1125 (1982).

6. E.V. Bakhanova, F.T.Vasko Dielectric constant of uniaxially compressed semiconductor with degenerate bands // Sov. Phys. Semicond. 21(6), pp.1068-1074 (1987).

7. E.V. Bakhanova, F.T. Vasko, Effect of crystal anisotropy on infrared hole transitions in uniaxially compressed semiconductors // Phys. stat.sol.(b), 182(1) pp. 97-107 (1994).

8. E.V. Bakhanova, F.T. Vasko, Infrared Raman scattering in uniaxially compressed semiconductors // Solid State Commun. 76(8) pp. 981-984 (1990). 


\section{V.N. Poroshin et al.: Stress-induced effects in light scattering by plasmons...}

9. A.V.Gaydar, V.N.Poroshin, Light scattering by plasmons in p-type germanium // Ukr. Fiz. Zh. 47 (9) pp. 862-866 (2002).

10. A.V.Gaydar, V.N.Poroshin, O.G.Sarbey, F.T.Vasko, Infrared light scattering by free holes in $p$-Ge // Semicond. Sci.Technol. 9(10), pp.1790-1794 (1994).

11. A. Dargys, J. Kundrotas, Handbook on physical properties of Ge, Si, GaAs, and InP, Science and Encyclopedia Publishers, Vilnius (1994).

12. V.N. Poroshin, A.V.Gaydar, Infrared Raman scattering by free holes in uniaxially compressed $p$-Ge // Ukr. Fiz. Zh. 48(5), pp.411-416 (2003).

13. G.A Abstreiter, M. Cardona, A. Pinczuk . Light Scattering in Solids, Eds. Springer-Verlag, Berlin-Heidelberg-New YorkTokio (1984).

14. I.V. Altukhov, M.S. Kagan, K.A. Korolev, V.N.Sinis, Intracenter inversion as a reason for the stimulated emission in uniaxially strained $p$-type germanium // JETP Letters. 59 (7), pp. 476-479 (1994).
15. A.T. Dalakyan, V.N. Tulupenko, D.A. Firsov, V.M. Bondar, On resonance states in split germanium // JETP Letters. 69 (9), pp. 676-681 (1999).

16. M.A. Odnoblyudov, I.N. Yassievich, V.M. Chistyakov, K.A. Chao, Resonant states induced by shallow acceptors in uniaxially strained semiconductors // Phys.Rev. 62(4). pp. 2486-2494 (2000)

17. A.A. Abramov, V.N. Poroshin, V.N. Tulupenko, Resonant impurity scattering in uniaxially stressed $p-G e / / U k r$. Fiz. Zh. 47(10), pp. 964-966 (2002)

18. M.A. Odnoblyudov, A.A. Pahomov, V.M. Chistyakov, I.N. Yassievich, Resonant acceptor states in uniaxially compressed semiconductors // Sov. Phys. Semicond. 31(10), pp. 1180-1186 (1997).

19. I.N. Yassievich, A.A Prokofuev, M.A. Odnoblyudov Resonant states and terahertz emission // Izvestiya RAN, ser. Fis. 65(2), pp. 240-242 (2001). 